\title{
24,2004
}

DRAFT. April

\section{Fitting Hyperbolic pants to a three-body problem.}

\begin{abstract}
Consider the three-body problem with an attractive $1 / r^{2}$ potential. Modulo symmetries, the dynamics of the bounded zero-angular momentum solutions is equivalent to a geodesic flow on the thrice-punctured sphere, or "pair of pants". The sphere is the shape sphere. The punctures are the binary collisions. The metric generating the geodesics is the Jacobi-Maupertuis metric. The metric is complete, has infinite area, and its ends, the neighborhoods of the punctures, are asymptotically cylindrical. Our main result is that when the three masses are equal then the metric has negative curvature everywhere except at two points (the Lagrange points). A corollary of this negativity is the uniqueness of the $1 / r^{2}$ figure eight, a complete symbolic dynamics for encoding the collision-free solutions, and the fact that collision solutions are dense within the bound solutions.
\end{abstract}

\section{Introduction and Results.}

We study the planar three-body problem with an attractive $1 / r^{2}$ potential. According to the Lagrange-Jacobi identity (eq. (3.7) below) every bounded solution must have zero energy and constant moment of interia $I$, and conversely, if an initial condition has zero energy and $\dot{I}(0)=0$ then that solution is bounded. Setting the moment of inertia $I$ equal to a constant defines a three-sphere in configuration space. Rotations act on this sphere according to the Hopf flow so that the quotient of the three-sphere by rotations is the two-sphere or shape sphere. See figure 1a. Points of this shape sphere represent oriented similarity classes of triangles. Newton's equations, for solutions with $H=0, \dot{I}=0$, push down to the shape sphere to yield a a family of second-order ODEs parameterized by the angular momentum. These ODEs have singularities at the three points representing the three types of binary collisions. Upon deleting the collision points we arrive at dynamics on the pair of pants, - the two-sphere minus three points. When the angular momentum is zero the resulting dynamical system is, after a time reparameterization, the geodesic flow for a certain Riemannian metric on the pair of pants. This metric is the (reduced) Jacobi-Maupertuis metric for energy 0.

Proposition 1. Endow the pair of pants with the Jacobi-Maupertuis metric (equations $(3.9 \mathrm{a}, \mathrm{b})$ below). Modulo rotations, translation, and scaling, the set of bounded zeroangular momentum solutions for the $1 / r^{2}$ potential three-body problem are in bijective correspondence with geodesics for this metric. The metric is complete and its ends (the deleted neighborhoods of the three binary collisions) are asymptotic to Euclidean cylinders of positive radii.

Proof: Section 3 .

See figure $1 b$ for a depiction of the pair of pants. 


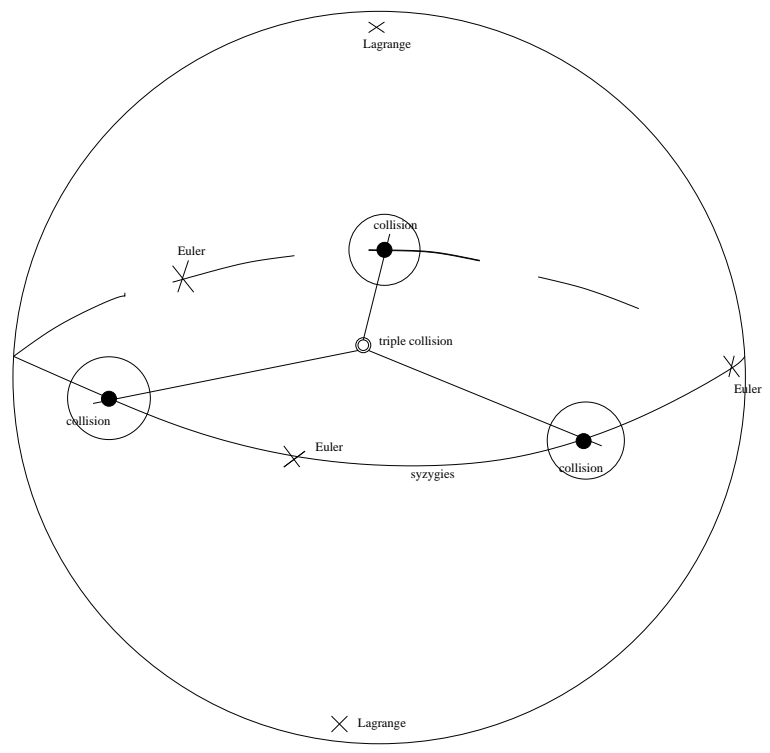

Figure 1a. The shape sphere

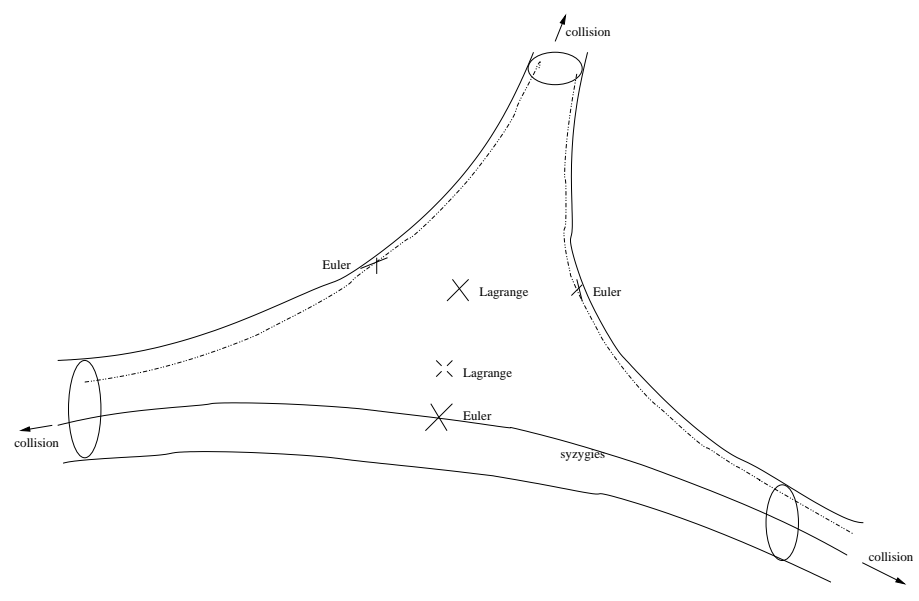

Figure 1b. The pair of pants

The Jacobi-Maupertuis metric depends parametrically on the masses of the three bodies by way of the potential (eq. 3.1). Our main result is:

Theorem 1. If all three masses are equal then the Gaussian curvature for the JacobiMaupertuis metric on the pair of pants is negative everywhere except at the two Lagrange points, where it is zero.

Proof. Section 4.

One might hope that negativity of the curvature persists for unequal masses. It does not. See section 7 . 


\section{Motivation and Dynamical Consequences.}

\subsection{Periodic Orbits and their symbol sequences.}

This work began as an attempt to give an analytic proof that the Newtonian $(1 / r$ potential) figure eight solution of Moore-Chenciner-Montgomery ([Moore], [ChMont]) is unique. I began with the easier case of the $1 / r^{2}$ eight. The figure eight is a periodic solution which realizes a certain free homotopy class on the pair of pants. Figure eights exists for all $1 / r^{a}$ potentials, $a>0$ ([CGMS], [FerrTerr]). For $a \geq 2$, not only is the free homotopy class of the eight realized, but almost every free homotopy class is realized by a solution. Combining these facts suggested the approach of this paper, and Theorem 1.

Our pair of pants metric from theorem 1 is neither compact, nor of negative curvature everywhere. But on a complete, noncompact surface of negative curvature if a free homotopy class has a geodesic representative, then that representative is unique. And uniqueness continues to hold if the curvature vanishes on a discrete set of points. (This theorem is fairly well-known, and proved in in a more general context in section 6.4 below, and in particular eq (6.4.3.).) We have proved

Corollary. For the $1 / r^{2}$ equal-mass zero-angular-momentum three-body problem, if a solution realizes a given free homotopy class on the pair of pants, then that solution is unique modulo rotation and scaling. In particular the eight is unique modulo these symmetries.

In stating the corollary we begged the question of which classes are realized. Every class is realized with the exception of those classes which wind around a single end. (See [MontN]). Gordon [1970] calls these 'bad' or unrealizable classes 'untied' while the complementary 'good', or realizable classes he called 'tied', being that they are 'tied' to the collision singularities. On the pants, a bad class can be represented by drawing a small circle, or "anklet" around one pants leg, and traversing it some number of times. As this "anklet" is pushed down towards the end of the leg its length decreases. As a result, any minimizing sequence of curves realizing such a class "falls off" of the leg. (See theorem 3 below.)

We follow $[\mathrm{MontN}]$ in using syzygies to describe the tied and untied classes. A syzygy is a collinear configuration of the three bodies. Syzygies come in three flavors, 1,2, and 3, depending on which mass is between the other two. (We exclude collisions.) The collinear configurations form the equator of the shape sphere. (Figure 1a.) The three collisions lie on the equator so that deleting them divides the equator into three arcs, again labelled $1,2,3$ according to the mass in the middle. A curve on the shape sphere has an associated syzygy sequence: list the syzygies in order. (Assume that the syzygy times are discrete.) The syzygy sequence of a motion of the three bodies is obtained by projecting the motion onto the shape sphere and writing out the syzygy sequence of the curve resulting on the shape sphere.

Periodic curves give rise to periodic sequences. For example, the class in which 1 and 2 circle about each other for ever while 3 remains far away has syzygy sequence . . 121212 ... (This is a "bad" class as we see later.) We subject syzygy sequences to the no stutterring rule: if $i j$ are consecutive letters of the sequence, then $i \neq j$. The reason for imposing this rule is that a stutter can be homotoped away. See figure 2 .

A letter $j$ with a plus superscript, as in $j^{+}$, denotes that syzygy $j$ occurs by crossing from the upper to the lower hemisphere of the sphere. A $j^{-}$means that syzygy $j$ occurs by 
crossing from the lower to the upper hemisphere of the sphere. Pluses and minuses must alternate, since the path will alternate between hemispheres. In topological terms an arc segment with two consecutive pluses, such as $k^{-} i^{+} j^{+}$lies entirely in the upper hemisphere and can be homotoped to $k^{-} j^{+}$. In dynamical terms such an arc can never occur since at $i^{+}$it would have to be tangent to the collinear subspace (the equator), but if a solution is tangent to the collinear subspace at a point then it lies completely within the collinear subspace.

It follows from the above considerations, and the topology of the pair of pants, that there is a one-to-one onto correspondence between free homotopy classes and periodic signed non-stuttering syzygy sequences. From now on we will drop the signing indications -the,+- superscripts - for simplicity. (Given an unsigned sequence there are only two ways to decorate it with signs.) The untied (unrealizable) classes are precisely those with

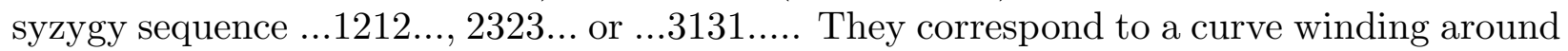
a single end. We prove in theorem 3 that there is no bounded zero angular momentum solution which realizes them. Excluding these classes is equivalent to insisting that all three letters occur in the sequence. Thus the corollary asserts that every periodic nonstuttering syzygy sequence in which all three letters occur is realized by a unique (up to symmetry) relative periodic solution.

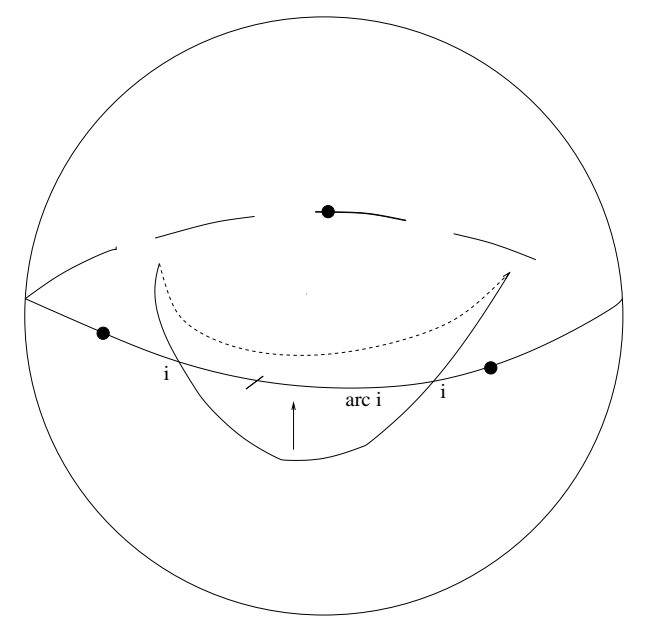

Figure 2. Homotoping away a stutter.

\subsection{Symbolic Dynamics; aperiodic syzygy sequences.}

We move on to infinite aperiodic syzygy sequences. In the rest of this subsection, "the problem' means the $1 / r^{2}$ equal-mass zero-angular-momentum three-body problem, and 'solution' means a solution to the problem, i.e. this differential equation. Many of the ideas and results here are adaptations of those pioneered in [Morse] and [Hadamard] XX Ref: use Had? .

Theorem 2. Every infinite nonstuttering syzygy sequence with the exception of the untied classes . . .ijij ... is realized by a solution. 
Proof. Section 6.2. The method is the classical one [Morse] of approximation by periodic solutions.

Theorem 3. If a syzygy sequence ends (begins) with ijij ... then any bounded solution which realizes this sequence must end (begin) in the $i j$ collision. The untied sequences $\ldots i j i j \ldots$ are not realized by any solution.

Proof. Section 6.3.

It is perhaps worth remarking that if a solution suffers collision then it does so in finite Newtonian time, but infinite "Jacobi time".

Inspired by theorem 3, we call the sequences appearing there "collision sequences". In more detail:

Definition. A bi-infinite nonstuttering syzygy sequence $s=\left\{s_{j}\right\}_{j=-\infty}^{+\infty}$ is a forward collision sequence if one of its forward tails $\left\{s_{j}\right\}_{j>N}$ contains only two letters. Similarly, we have backward collision sequences. A collision sequence is one which is either forward or backward collision sequence. In the contrary case, all three letters occur in every tail, and the sequence is called collision-free.

Does every solution have a syzygy sequence? If so, is this sequence unique? In [MontI] I showed that every bounded noncollinear zero-angular momentum solution to the Newtonian three-body problem suffers infinitely many syzygies, provided the solution does not tend to triple collision, and provided binary collisions are counted as syzygies. (See [Fuji] for another proof.) That proof works verbatim for any $1 / r^{a}$ potential, $a>0$, with the exception that we must exclude binary collisions. (They cannot be regularized.) Thus every bounded collision-free solution has a syzygy sequence. That sequence must be nonstuttering in our equal mass case. To prove that there is no stuttering, use the fact that on a surface of negative curvature any compact geodesic arc is the unique length minimizing curve among all homotopic curves which share its endpoints. (See equation 6.3 and its derivation.) Consequently, an application of the method of reflection as exposed in $[\mathrm{ChM}]$ rids us of solution arcs representing stutters, i.e. solution arcs which hit the same equatorial arc twice in a row. These considerations allow us to define a syzygy map from collision-free sequences to infinite nonstuttering syzygy sequences.

Theorem 4. The syzygy map from bounded solutions to syzygy sequences is a bijection between the set of collision-free solutions, modulo symmetry and time-translation, and the set of bi-infinite nonstuttering collision-free syzygy sequences, modulo shift.

Proof. Section 6.4.

Finally, we would like to know how much of phase space (the unit tangent bundle of the pair of pants) is taken up by the noncollision solutions. Not much:

Theorem 5. Solutions tending to binary collision are dense within the space of all bounded solutions. Thus the collision-free solutions have empty interior.

Proof. Section 6.5.

Summary. Putting the theorems together gives a rather complete symbolic dynamical picture of the dyanmics of our problem - the zero-angular momentum equal-mass $1 / r^{2}$ 
three body problem restricted to the bound orbits - those with $I=$ const.. There are no linearly stable periodic orbits, by theorem 1. We will use the word "bounded" in the rest of this paragraph to mean solutions which tend to to collision, as these orbits are precisely the geodesics on the pair of pants which tend to infinity.

Theorem 4 provides a complete symbolic dynamics picture for the bound orbits : they are precisely the orbits none of whose tails agree with the tied sequences ...ijij .... The closure of this set of orbits is the recurrent set. The recurrence set also coincides with the closure of the set of periodic orbits. There are unbounded orbits on the frontier of this closure. The situation is similar to that of the recurrent set for the Kepler problem: the space of periodic orbits is the recurrent set and contains the unbounded parabolic orbits. These unbounded recurrent orbits are "just barely unbound" in that the collision condition $J_{1}^{2}-\left(m_{1}+m_{2}\right) \leq 0$ occuring in the appendix A, inequality (12A) is an equality on these orbits. The complement of the recurrent set consists of orbits tending "strongly" to a binary collision. These strongly colliding orbits form an open set. (Appendix A.) Finally, the density result, theorem 6 , is an analogue of what one would like to prove for the honest $1 / r$ three-body problem: that the set of solutions tending to infinity (via tight binary pairs) is dense, for fixed energy and angular momentum. M. Hermann calls this density question "the oldest problem in dynamical systems [Hermann] XX.

Loose ends. There are some collision orbits which we have left out of symbol sequence considerations. The collinear solutions are not accounted for. (Collinear solutions should either have no syzygy sequence or a continuum of ' $i$ 's as their sequence, depending on one's taste.) There are exactly six collinear solutions, two for each of the three collision arcs, the two being related by reversing orientation. There are also collision orbits which end in collision but without the bodies winding around infinitely often. They 'head straight in' to infinity down one of the pants legs and their corresponding syzygy sequences will truncate in the forward direction, for forward time collision. The simplest of these truncated solutions are the isosceles solutions. Again, there are six of these by the same counting as for colliner solutions. The isosceles solution $r_{i j}=r_{i k}$ begins and ends at the $j k$ collision, and has exactly one syzygy in between, the Euler point in which $i$ is at the midpoint of $j$ and $k$. Its syzygy sequence is the single letter ' $i$ '. Interpolating between collinear and isosceles is a one-parameter family of solutions whose syzygy sequences truncate. These interpolating solutions are the $\lambda$-curves of eq. (3.13), the curves of constant $\chi$, in the $\lambda, \chi$ coordinate system there. I do not know if the syzygy sequences of these solutions are finite, or one-sided infinite.

Open questions. 1. Can two distinct collision orbits share the same syzygy sequence?

2. Are there any solutions besides isosceles which have a finite syzygy sequence? If so, can any finite syzygy sequence occur as the syzygy sequence of some collision orbit? 


\section{Set-Up and Proof of Prop. 1}

Write $x=\left(x_{1}, x_{2}, x_{3}\right) \in \mathbb{R}^{6}$ with $x_{i} \in \mathbb{R}^{2}$ for the positions of the three bodies, and $r_{i j}=\left\|x_{i}-x_{j}\right\|$ for the distances between them. The potential is $-U$ where

$$
U=\Sigma m_{i} m_{j} / r_{i j}^{2}
$$

The $m_{i}$ are the masses. Set

$$
\begin{aligned}
K & =\Sigma m_{i}\left\|\dot{x}_{i}\right\|^{2} \\
& =\langle\dot{x}, \dot{x}\rangle,
\end{aligned}
$$

for twice the kinetic energy. The last equality of (3.2) defines the "mass inner product" on the three-body configuration space $\mathbb{R}^{6}$. The total energy

$$
H=K / 2-U
$$

is constant along solutions. The equations of motion, $\ddot{x}_{i}=-2 \Sigma_{i \neq j} m_{j}\left(x_{i}-x_{j}\right) / r_{i j}^{4}, i=$ $1,2,3$, can be written as the single vector equation

$$
\ddot{x}=\nabla U
$$

where $\nabla U$ is defined using the mass inner-product: $d U(x)(v)=\langle\nabla U(x), v\rangle$.

By the standard method of freshman physics, we can, without loss of generality restrict our considerations to motions for which

$$
\Sigma m_{i} x_{i}=0
$$

throughout. This constraint defines a four-dimensional real vector space which can be identified with the two-dimensional complex space $\mathbb{C}^{2}$ in such a way that counterclockwise rotation of a triangle $\left(x_{1}, x_{2}, x_{3}\right)$ by $\theta$ radians turns into scalar multiplication of the corresponding complex vector by $\exp (i \theta)$. Set

$$
\begin{aligned}
I & =\Sigma m_{i} m_{j} r_{i j}^{2} / \Sigma m_{i} \\
& =\langle x, x\rangle
\end{aligned}
$$

where the last equality is only true when the center of mass constraint (3.5) is in place. Using $\dot{I}=2\langle x, \dot{x}\rangle, \ddot{I}=2\langle\dot{x}, \dot{x}\rangle+2\langle x, \ddot{x}\rangle$ and $\langle x, \nabla U(x)\rangle=-2 U(x)$ (by $U$ 's homogeneity) we obtain the Lagrange-Jacobi identity:

$$
\ddot{I}=4 H
$$

valid along any solution. Thus $I(t)=$ const. along the solution if and only if $H=0$ and $\dot{I}(0)=0$ for that solution.

We will call a solution "bounded" if the $r_{i j}$ are bounded as functions of time, and do not simultaneously tend to zero, i.e. to triple collison. Now $I \rightarrow \infty$ if and only if one of the $r_{i j}$ tend to infinity, and $I \rightarrow 0$ if all $r_{i j} \rightarrow 0$. It follows from (3.7) that every bounded solution must satisfy $H=0, \dot{I}(0)=0$, and $I(t)=$ const. 
The scaling symmetry $x(t) \mapsto \lambda^{-1 / 2} x(\lambda t)$ takes solutions to solutions, preserves zero energy, and takes $I$ to $I / \lambda$. Using this scaling, we may, without loss of generality, assume that $I=1$ in studying bounded solutions. The set $I=1, \Sigma m_{i} x_{i}=0$ forms a three-sphere in the $\mathbb{C}^{2}$. We have reduced the study of the bounded solutions to the $1 / r^{2}$ problem to a second order dynamics on this three-sphere. It is well-known ([Arn] or [AbMar]) that a dynamics on a constant energy surface $H=E$ level set is equivalent to geodesic flow for the Jacobi-Maupertuis metric $(E+U) d s^{2}$ where $d s^{2}$ is the kinetic energy metric. In our case, $E=0$, and we restrict the kinetic energy to the sphere $I=1$. Consequently, the study of bounded solutions is equivalent to the study of geodesics on the three-sphere under the metric $d s_{J}^{2}=U d s^{2}$ conformal to the standard metric $d s^{2}$ on that three-sphere.

To obtain a metric on the shape sphere, we quotient by rotations. We review the discussion in [MontN], [ChMont], or [MontR] on this metric. See especially the appendix of [MontR] for explicit computations and derivations. The group of rigid rotations acts on the three-body configuration space according to scalar multiplication on $\mathbb{C}^{2}$ by unit modulus complex scalars. Restricting ourselves to the three-sphere $S^{3}:=\{I=1\} \subset \mathbb{C}^{2}$ and forming the quotient by this rotational action yields the famous Hopf fibration

$$
S^{3} \rightarrow S^{2}=S^{3} / S^{1}
$$

The quotient two-sphere is the shape sphere ([ChM], $[$ MontR $]$ esp. the appendix). Its points represent oriented similarity classes of triangles. Both the dynamics and the Jacobi metric $U d s^{2}$ on the three-sphere descend under the projection (3.8) to the shape sphere once we fix the value of the total angular momentum. The total angular momentum of a solution is zero if and only if that solution is orthogonal to the rotational orbits, i.e. orthogonal to the fibers of (3.8). The projection of such a zero-angular momentum solution under (3.8) is a geodesic for the quotient metric. (See [Hermann], lemma 4.1. His situation is more general than ours. Our circle bundle (3.8) is replaced by a general Riemannian submersion.) We can write the Jacobi-Maupertuis metric on the shape sphere as

$$
d s_{J}^{2}=U d s_{\text {shape }}^{2}
$$

where $d s_{\text {shape }}^{2}$ is the kinetic-energy induced metric on shape sphere, and $U$ is the (negative) potential (3.1) restricted to $I=1$ and then viewed as a fucntion on the shape-sphere (possible because of its rotation invariance. The shape sphere metric is the round metric on a sphere of radius $1 / 2$ :

$$
\left.d s_{\text {shape }}^{2}=\left(\frac{1}{2}\right)^{2}\left[d \phi^{2}+\cos ^{2}(\phi) d \theta^{2}\right)\right]
$$

where $\phi$ is the colatitude - the angle from the equator, and $\theta$ is labels longitudinal circles on the sphere.

Proof of Proposition 1. The discussion of the last two paragraphs shows that, modulo rotations, translation, and scaling, the set of bounded zero-angular momentum solutions for the negative potential (3.1) are in bijective correspondence with geodesics for the 
Jacobi-Maupertuis metric on the shape sphere minus the three binary collision. Under this correspondence the geodesic flow for the metric corresponds, after a time reparameterization, to the the flow defined by Newton's equations. It remains to verify the claims about the completeness and that the ends asymptote to cylinders.

\section{Completeness.}

Let $\rho=\rho_{i j}$ be the spherical distance from the $i j$ collision point $C_{i j}$, as measured in the spherical metric $d s_{\text {shape }}^{2}$. Then as $\rho \rightarrow 0$ we will show that

$$
U=\frac{C^{2}}{\rho^{2}}+O(1)
$$

for some positive constant $C^{2}$, while

$$
d s_{\text {shape }}^{2}=d \rho^{2}+\left(\rho^{2}+O\left(\rho^{4}\right)\right) d \chi^{2}
$$

where $\chi$ is the angular coordinate based at $\Sigma_{i j}$ so that $(\rho, \chi)$ are geometric polar coordinates. It follows that the Jacobi metric has the expansion:

$$
\left.d s_{J}^{2}=\frac{C^{2}}{\rho^{2}}\left(d \rho^{2}+\rho^{2} d \chi^{2}\right)\right)+O(1)
$$

It follows that if we approach the collision $\rho=0$ along any curve then the length of that curve diverges at least as fast as the integral of $C \sqrt{d \rho^{2} / \rho^{2}}=C d \rho / \rho$, that is, it diverges logarithmically as $C|\log (\rho)|$ as $\rho \rightarrow 0$. Consequently any curve tending towards "infinity" i.e. to one of the binary collisions, has infinite length, which proves completeness.

To establish (3.10a), it suffices to establish

$$
r_{i j}=\frac{1}{\sqrt{\mu_{i j}}} \sin \left(\rho_{i j}\right)
$$

where $\mu_{i j}=m_{i} m_{j} /\left(m_{i}+m_{j}\right)$ is the reduced mass. The other two distances $r_{i k}, r_{j k}$ are bounded away from zero as $r_{i j} \rightarrow 0$, due to the constraint $I=1$ (see (3.6)). Then (3.10a) follows from (3.1) and the Taylor expansion of $\sin (\rho)$. The constant $C$ in (3.10a) is $m_{i} m_{j} / \sqrt{\mu_{i j}}$.

To establish (3.11) we work in the full three dimensional shape space which is the space whose points are oriented congruence classes of planar triangles. The full shape space is isometric to the cone over the shape sphere and consequently distances $d$ in the full shape space can be obtained from spherical distances together with knowldge of the distance $R=\sqrt{I}$ from the cone point. Write $d_{i j}$ for the distance in the full shape space between an arbitrary point and the $i j$ binary collision ray. Then we have

$$
r_{i j}=\frac{1}{\sqrt{\mu_{i j}}} d_{i j}
$$

and

$$
d_{i j}=R \sin \left(\rho_{i j}\right)
$$


Upon setting $R=1,(3.11)$ follows immediately. Equation (3.12a,b) can be found in section 4 , equations $(4.3 .15 \mathrm{a}, \mathrm{b})$ of [MontN]. However, note that there is a typo in eq 4.3.15a. The $\mu_{i j}$ in that equation must be replaced by $\sqrt{\mu_{i j}}$.)

To get (3.10b) use the fact that the shape sphere is isometric to the sphere of radius $1 / 2$ and that the metric on such a sphere is given by

$$
\left.d s_{\text {shape }}^{2}=d \rho^{2}+[(1 / 2) \sin (2 \rho))\right]^{2} d \chi^{2}
$$

in spherical-polar coordinates. Then use the Taylor expansion of $(1 / 2) \sin (2 \rho)$.

Asymptotes to Cylinders. We use a more precise version of the expansion (3.10c). Set

$$
d \lambda=-\sqrt{U} d \rho
$$

Integrating (3.14) defines a function $\lambda=\lambda(\rho, \chi)$ such thta $\lambda \rightarrow \infty$ as the collision $\rho=0$ is approached. From (3.10a) we have $d \lambda=-C d \rho / \rho+O(1)$ from which it follows that

$$
\rho=e^{-C \lambda}+o(\rho) \text {. }
$$

The $(\lambda, \chi)$ are coordinates for the end $\rho=0$, and in these coordinates

$$
d \bar{s}^{2}=d \lambda^{2}+f(\lambda, \chi)^{2} d \chi^{2}
$$

where, from (3.13) and (3.9a) we have

$$
f^{2}=\left(\frac{1}{2} \sin (\rho)\right)^{2} U
$$

Now $\left(\frac{1}{2} \sin (2 \rho)\right)^{2}=\sin ^{2} \rho \cos ^{2} \rho$ so that from $(3.11)\left(\frac{1}{2} \sin (2 \rho)\right)^{2}=\mu_{i j} r_{i j}^{2} \cos ^{2}(\rho)$ and

$$
f^{2}=\mu_{i j} \cos ^{2}(\rho)\left\{m_{i} m_{j}+m_{i} m_{k} \frac{r_{i j}^{2}}{r_{i k}^{2}}+m_{j} m_{k} \frac{r_{i j}^{2}}{r_{j k}^{2}}\right\}
$$

where $i j k$ is a permutation of 123. As we approach the collision $\lambda=\infty$ we have $r_{i j} \rightarrow 0$ while $r_{i k}, r_{j k}$ remain bounded since we are constrained to $I=1$. Thus

$$
\lim _{\lambda \rightarrow \infty} f=\sqrt{\mu_{i j} m_{i} m_{j}}:=K_{i j}>0
$$

Summarizing:

$$
d s_{J}^{2}=d \lambda^{2}+\left(K_{i j}^{2}+O\left(e^{-2 C \lambda}\right)\right) d \chi^{2}
$$

which says the metric asymptotes to a Euclidean cylinder of radius $K_{i j}$ as we approach the $i j$ end.

QED

Remark. It follows from equations $(7.12, .13)$ that the Gaussian curvature near the end is negative. But for any metric of the form (3.15) this curvature is equal to $-\frac{1}{f} \frac{\partial^{2} f}{\partial \lambda^{2}}$. Thus, 
for fixed $\chi$, the function $f(\lambda, \chi)$ is a strictly convex of $\lambda$, for all $\lambda$ from some point on, and from this point on, $f(\lambda, \chi)$ monotonically decreases to $K_{i j}$.

\section{Curvature. Proof of theorem 1.}

We proceed to the proof of our main result, Theorem 1, the negativity of the Gaussian curvature when the masses are equal. The computation proceeds through a series of lemmas. The first is standard and we will not provide the proof.

Lemma 4.1. Let a surface be endowed with conformally related metrics $d s^{2}$ and $d \bar{s}^{2}=$ $U d s^{2}$. Then their curvatures $K, \bar{K}$ are related by

$$
\bar{K}=U^{-1}\left(K-\frac{1}{2} \Delta \log (U)\right)
$$

where the Laplacian $\Delta$ is with respect to the $d s^{2}$ metric.

The curvature $K$ of the standard shape metric $d s^{2}=d s_{\text {shape }}^{2}$ of $(3.9 \mathrm{~b})$ is $K=4$. According to lemma 4.1

$$
\bar{K}=4-\frac{1}{2} \Delta(\log (U)
$$

is the desired curvature, the curvature of the Jacobi-Maupertuis metric (3.9a) of proposition 1 and Theorem 1. A routine computation yields

$$
\Delta(\log (U))=\frac{U \Delta U-\|\nabla U\|^{2}}{U^{2}}
$$

Here, and throughout this section, $U$ is considered as a function on the shape sphere, $\Delta U$ is its Laplacian with respect to the standard shape space metric metric $d s^{2}$ and $\|\nabla U\|^{2}$ is the squared length of its gradient with respect to the same metric.

A key to the subsequent computations is to use the squared length coordinates as in [AlbCh]

$$
s_{k}=r_{i j}^{2}, i j k \text { a permutation of } 123,
$$

rather than the lengths $r_{i j}$ themselves. Write

$$
U_{2 n}=\Sigma 1 / r_{i j}^{2 n}=\Sigma 1 / s_{k}^{n}
$$

so that $U=U_{2}$.

\section{Lemma 2.}

$$
\Delta U=8 U_{4}
$$

Proof. Section 5.2. 


\section{Lemma 3.}

$$
\|\nabla U\|^{2}=4 S
$$

where

$$
S=2 U_{6}-U_{4}-3 / 2 \Sigma^{\prime} 1 / s_{i}^{2} s_{j}^{2}+2 \Sigma^{\prime} 1 / s_{i} s_{j}^{2}-\Sigma^{\prime} 1 / s_{i} s_{j}
$$

and where ' $\Sigma$ '" means to sum over all indices $i, j$ with $i \neq j$. (For example $\Sigma$ ' $s_{i} s_{j}=$ $2 s_{1} s_{2}+2 s_{2} s_{3}+2 s_{3} s_{1}$, twice the second symmetric polynomial in the $s_{i}$.)

Proof. Section 5.3.

Proof of the Negativity of the curvature. Combining the equations (4.1), (4.2), (4.5) and (4.6a) we find

$$
-\bar{K} U^{3}=4 U U_{4}-4 U^{2}-2 S
$$

Expand out the first two terms on the right hand side:

$$
\begin{aligned}
U U_{4} & =\Sigma 1 / s_{i} \Sigma 1 / s_{j}^{2} \\
& =\Sigma 1 / s_{i}^{3}+\Sigma^{\prime} 1 / s_{i} s_{j}^{2} \\
& =U_{6}+\Sigma^{\prime} 1 / s_{i} s_{j}^{2} .
\end{aligned}
$$

while

$$
\begin{aligned}
U^{2} & =\Sigma 1 / s_{i} \Sigma 1 / s_{j} \\
& =\Sigma 1 / s_{i}^{2}+\Sigma^{\prime} 1 / s_{i} s_{j} \\
& =U_{4}+\Sigma^{\prime} 1 / s_{i} s_{j} .
\end{aligned}
$$

Plugging (4.8), (4.9) and equation (4.6b) back into equation (4.7) yields :

$$
-\bar{K} U^{3}=-2 U_{4}-2 \Sigma^{\prime} 1 / s_{i} s_{j}+3 \Sigma^{\prime} 1 / s_{i}^{2} s_{j}^{2}
$$

Use the fact that $U_{4}+\Sigma^{\prime} 1 / s_{i} s_{j}=\left(\Sigma 1 / s_{i}\right)^{2}=U^{2}$ to rewrite the right hand side of (4.10), and divide the resulting equation by two in order to obtain

$$
-\bar{K} U^{3}=3 \Sigma^{\prime} 1 / s_{i}^{2} s_{j}^{2}-2 U^{2}
$$

Consequently, $\bar{K} \leq 0$ if and only if

$$
3\left(\Sigma^{\prime} 1 / s_{i}^{2} s_{j}^{2}\right) \geq 2\left(\Sigma 1 / s_{i}\right)^{2}
$$

To prove (4.11), multiply both sides of it by $s_{1}^{2} s_{2}^{3} s_{3}^{2}$ thus arriving at $6\left(\Sigma s_{i}^{2}\right) \geq 2 \sigma_{2}^{2}$ or

$$
3\left(\Sigma s_{i}^{2}\right) \geq \sigma_{2}^{2}
$$

where $\sigma_{2}=s_{1} s_{2}+s_{2} s_{3}+s_{3} s_{1}$ is the second elementary symmetric polynomial in the $s_{i}$. (The coefficient 6 arose because for each pair $i j$ there are two terms in the sum $\Sigma^{\prime}$. See the parenthetical remark in lemma 3.) To prove (4.12), remember that we are restricting 
ourselves to the sphere $I=1$ and that $I=\Sigma s_{i} / 3$. Thus we can homogenize the equation by using that $3=\left(\Sigma s_{i}\right)^{2} / 3$ on the sphere. So, the desired inequality now reads:

$$
\frac{\left(\Sigma s_{i}\right)^{2}}{3}\left(\Sigma s_{i}^{2}\right) \geq \sigma_{2}^{2}
$$

The two inequalities:

$$
\Sigma s_{i}^{2} \geq \sigma_{2}
$$

and

$$
\frac{\left(\Sigma s_{i}\right)^{2}}{3} \geq \sigma_{2}
$$

are classical, with equality in either case if and only if all the $s_{i}$ are equal. Here are the proofs. Inequality $(4.13 \mathrm{~A})$ follows simply upon rearranging the inequality $\left(s_{1}-s_{2}\right)^{2}+$ $\left(s_{2}-s_{3}\right)^{2}+\left(s_{3}-s_{1}\right)^{2} \geq 0$. Inequality (4.13B) is a special case of a general inequality among the elementary symmetric polynomials evaluated at positive arguments $s_{i}$. See for example the Encyclopaedia [Math], App. A, Table 8, inequality (4). Alternatively, expand out $\left(\Sigma s_{i}\right)^{2}=\Sigma s_{i}^{2}+2 \sigma_{2}$ and use inequality (4.13A). Multiplying the two inequalities yields the desired inequality (4.12), with equality if and only if we are at the Lagrange points $s_{1}=s_{2}=s_{3}$ of the shape sphere. Since (4.12) is equivalent to the curvature inequality, Theorem 1 is proved, modulo the proofs of lemmas 2 and 3 which follow in the next section. QED

\section{Proofs of lemmas 2 and 3.}

\subsection{Notation.}

To compute $\Delta U$ and $\|\nabla U\|^{2}$ we must express the squared distances $s_{k}=r_{i j}^{2}$ of (4.3) in terms of the spherical coordinates $(\phi, \theta)$ of $(3.9 \mathrm{~b})$. In [MontI] I prove that upon restriction to the sphere $I=1$

$$
s_{k}=1-\cos (\phi) \gamma_{k}(\theta)
$$

where

$$
\gamma_{1}(\theta)=\cos (\theta), \gamma_{2}(\theta)=\cos (\theta+2 \pi / 3), \gamma_{3}(\theta)=\cos (\theta+4 \pi / 3)
$$

The special angles $\theta=0,2 \pi / 3,4 \pi / 3$ mark the locations of the three binary collision on the equator $\phi=0$ of collinear triangles. Later on we will use the fact that the three planar vectors $\left(\gamma_{k}, \gamma_{k}^{\prime}\right), k=1,2,3$ form the vertices of an equilateral triangle inscribed in the unit circle. Here and throughout we write $\gamma_{i}^{\prime}$ for the derivative $\partial_{\theta} \gamma_{i}$ of $\gamma_{i}$ with respect to $\theta$.

5.2. Proof of Lemma 2. Write $c=\cos (\phi), \partial_{\phi}$ for the partial derivative with respect to $\phi$ and $\partial_{\theta}$ for the partial derivative with respect to $\theta$. Then

$$
\Delta U=\frac{4}{c} \partial_{\phi}\left(c \partial_{\phi} U\right)+\frac{4}{c^{2}}\left(\partial_{\theta}^{2} U\right)
$$


And

$$
\begin{gathered}
\partial_{\phi}\left(1 / s_{i}\right)=-s \gamma_{i} / s_{i}^{2} \\
\partial_{\theta}\left(1 / s_{i}\right)=c \gamma_{i}^{\prime} / s_{i}^{2}
\end{gathered}
$$

Since $U=\Sigma 1 / s_{i}$ we have

$$
\begin{aligned}
\partial_{\phi}\left(c \partial_{\phi} U\right) & =\partial_{\phi} c \Sigma\left(-s \gamma_{i}\right) / s_{i}^{2} \\
& =\partial_{\phi}\left(-c s \Sigma \gamma_{i}\right) / s_{i}^{2} \\
& =\left(-c^{2}+s^{2}\right) \Sigma \gamma_{i} / s_{i}^{2}+2 c s^{2} \Sigma \gamma_{i}^{2} / s_{i}^{3}
\end{aligned} .
$$

Thus

$$
\frac{1}{c} \partial_{\phi}\left(c \partial_{\phi} U\right)=\left(\left(s^{2}-c^{2}\right) / c\right) \Sigma \gamma_{i} / s_{i}^{2}+2 s^{2} \Sigma \gamma_{i}^{2} / s_{i}^{3} .
$$

And

$$
\begin{aligned}
\frac{1}{c^{2}} \partial_{\theta} \partial_{\theta} U & =\frac{1}{c^{2}} \partial_{\theta} \Sigma c \gamma_{i}^{\prime} / s_{i}^{2} \\
& =\frac{1}{c^{2}} \Sigma c\left[\gamma_{i}^{\prime \prime} / s_{i}^{2}+2\left(c \gamma_{i}\right)^{2} / s_{i}^{3}\right. \\
& =\frac{1}{c} \Sigma \gamma_{i}^{\prime \prime} / s_{i}^{2}+2 \Sigma\left(\gamma_{i}^{\prime}\right)^{2} / s_{i}^{3}
\end{aligned}
$$

Now $\gamma_{i}^{\prime \prime}=-\gamma_{i}$ so that

$$
\frac{1}{c^{2}} \partial_{\theta} \partial_{\theta} U=-\frac{1}{c} \Sigma \gamma_{i} / s_{i}^{2}+2 \Sigma\left(\gamma_{i}^{\prime}\right)^{2} / s_{i}^{3}
$$

Adding 4 times (5.2.4) to 4 times (5.2.5) we get

$$
\Delta U=4\left(\left(s^{2}-c^{2}-1\right) / c\right) \Sigma \gamma_{i} / s_{i}^{2}+8 s^{2} \Sigma \gamma_{i}^{2} / s_{i}^{3}+8 \Sigma\left(\gamma_{i}^{\prime}\right)^{2} / s_{i}^{3}
$$

Now $s^{2}-c^{2}-1=-2 c^{2}$ so that

$$
\Delta U=-8 \Sigma c \gamma_{i} / s_{i}^{2}+8 \Sigma\left[s^{2} \gamma_{i}^{2}+\left(\gamma_{i}^{\prime}\right)^{2}\right] / s_{i}^{3}
$$

Recalling that $\gamma_{i}^{2}+\left(\gamma_{i}^{\prime}\right)^{2}=1$ (the vectors $\left(\gamma_{i}, \gamma_{i}^{\prime}\right)$ define an equilateral triangle inscribed in the unit circle) we see that we can replace $\left(\gamma_{i}^{\prime}\right)^{2}$ by $1-\gamma_{i}^{2}$ in order to obtain

$$
\begin{aligned}
s^{2} \gamma_{i}^{2}+\left(\gamma_{i}^{\prime}\right)^{2} & =\left(s^{2}-1\right) \gamma_{i}^{2}+1 \\
& =-c^{2} \gamma_{i}^{2}+1 \\
& =-\left(1-s_{i}\right)^{2}+1 \\
& =2 s_{i}-s_{i}^{2} \\
& =s_{i}\left(2-s_{i}\right)
\end{aligned}
$$

Then $\left[s^{2} \gamma_{i}^{2}+\left(\gamma_{i}^{\prime}\right)^{2}\right] / s_{i}^{3}=\left(2-s_{i}\right) / s_{i}^{2}=\left(c \gamma_{i}+1\right) / s_{i}^{2}$ where I used $1-s_{i}=c \gamma_{i}$ It follows that

$$
\Delta U=-8 \Sigma c \gamma_{i} / s_{i}^{2}+8 \Sigma c \gamma_{i} / s_{i}^{2}+8 \Sigma\left(1 / s_{i}^{2}\right)=8 U_{4}
$$


as claimed.

\subsection{Proof of Lemma 3.}

We have

$$
\begin{aligned}
d U & =\partial_{\phi} U d \phi+\partial_{\theta} U d \theta \\
& =\left(\Sigma\left(-s \gamma_{i} / s_{i}^{2}\right) d \phi+\left(\Sigma\left(c \gamma_{i}^{\prime}\right) / s_{i}^{2}\right)\right) d \theta
\end{aligned}
$$

Now $\|\nabla U\|^{2}=\|d U\|^{2}$. The length squared of the covector $d U$ is computed relative to the metric ' $g^{i j}$ ' induced on covectors, which, from (3.9) is given at the point $(\phi, \theta)$ by $\|a d \phi+b d \theta\|^{2}=4\left(a^{2}+\frac{1}{c^{2}} b^{2}\right)$, with $c=\cos (\phi)$. It follows that

$$
\begin{aligned}
\|\nabla U\|^{2} & =4\left(\Sigma s \gamma_{i} / s_{i}^{2}\right)^{2}+4\left(\Sigma \gamma_{i}^{\prime} / s_{i}^{2}\right)^{2} \\
& =4\left(\Sigma s^{2} \gamma_{i}^{2} / s_{i}^{4}+\Sigma^{\prime} s \gamma_{i} s \gamma_{j} / s_{i}^{2} s_{j}^{2}+\Sigma \gamma_{i}^{\prime 2} / s_{i}^{4}+\Sigma^{\prime} \gamma_{i}^{\prime} \gamma_{j}^{\prime} / s_{i}^{2} s_{j}^{2}\right) \\
& =4\left(\Sigma\left(s^{2} \gamma_{i}^{2}+\left(\gamma_{i}^{\prime 2}\right) / s_{i}^{4}+\Sigma^{\prime}\left(s^{2} \gamma_{i} \gamma_{j}+\gamma_{i}^{\prime} \gamma_{j}^{\prime}\right) / s_{i}^{2} s_{j}^{2}\right) .\right.
\end{aligned}
$$

Simplify the numerator in the first summand of the last equation by using (5.2.7). We will simplify the numerator of the second summand by using an analogous identity for $s^{2} \gamma_{i} \gamma_{j}+\gamma_{i}^{\prime} \gamma_{j}^{\prime}, i \neq j$. Indeed, since the vectors $\left(\gamma_{i}, \gamma_{i}^{\prime}\right)$ form the vertices of an equilateral triangle inscribed within the unit circle, we have that $\gamma_{i} \gamma_{j}+\gamma_{i}^{\prime} \gamma_{j}^{\prime}=-1 / 2$ for $i \neq j$, since $-1 / 2=\cos (2 \pi / 3)$ is the cosine of the central angle defined by any two vertices of an equilateral triangle. Thus

$$
\begin{aligned}
s^{2} \gamma_{i} \gamma_{j}+\gamma_{i}^{\prime} \gamma_{j}^{\prime} & =\gamma_{i} \gamma_{j}+\gamma_{i}^{\prime} \gamma_{j}^{\prime}-c^{2} \gamma_{i} \gamma_{j} \\
& =-1 / 2-\left(1-s_{i}\right)\left(1-s_{j}\right) \\
& =-3 / 2+s_{i}+s_{j}-s_{i} s_{j}
\end{aligned} .
$$

Plugging (5.3.2) and (5.3.3) into (5.3.1) we get

$$
\begin{aligned}
\|\nabla U\|^{2} & =4\left(2 \Sigma 1 / s_{i}^{3}-\Sigma 1 / s_{i}^{2}-\frac{3}{2} \Sigma^{\prime} 1 / s_{i}^{2} s_{j}^{2}+2 \Sigma^{\prime} 1 / s_{i} s_{j}^{2}-\Sigma^{\prime} 1 / s_{i} s_{j}\right) \\
& =4 S
\end{aligned}
$$

as claimed. QED

\section{Dynamical Consequences.}

\section{1}

Let $P$ denote the pair of pants. Using the spherical shape metric $(3.9 \mathrm{a})$, construct three disjoint circles with centers at the three binary collision points. Delete the open discs bounded by these circles to obtain a compact region $R \subset P$ having for its boundary the three disjoint circles. Refer back to figure 1.

Lemma 6.1. Any arc in $P$ whose (finite) syzygy sequence contains all three letters must cut through $R$.

Proof. Let $c$ be such a ' 123 ' arc. If one of $c$ 's syzygies is within $R$, we are done. Otherwise, all three syzygies lie within the three excised discs. But all three syzygies 
cannot be in the same disc, since each disc contains exactly two syzygy types. Thus $c$ must must travel from one disc to the other, and in so doing it cross into $R$.

QED.

6.2. Proof of Theorem 2. Let $s$ be syzygy sequence containing all three letters. Approximate $s$ by a sequence $w^{N}, N=1,2,3$ of periodic sequences as follows. Truncate $s$ to form the finite even length subword $w_{N}=s_{-N+1} s_{-N+1} \ldots s_{N}$. Turn this subword into a periodic sequence $\ldots w_{N} w_{N} w_{N} \ldots$ by repeating it in blocks. If the resulting word has stutters at the join, shift the "window" we used to form $w^{N}$ so as to form the word $w_{N, j}=s_{-N+j+1} s_{-N+j} \ldots s_{N+j}$ along with its corresponding periodic word. We can always find a $j$ so that the resulting periodic word, call it $w^{N}$, is non-stuttering. The sequence $w_{N}$ contain all three letters 123 for all $N$ sufficiently large, since $s$ itself contains all three letters. Theorem 2 implies that the $w_{N}$, for $N$ large, are represented by a unique geodesic $\gamma_{N}$. By lemma 6.1 the $\gamma_{N}$ must cut through $R$. Shifting time (and thus shifting the sequence) if necessary, we may assume that $\gamma_{N}(0) \in R$. Since $R$ is compact, so is the unit tangent bundle to $P$ (relative to the Jacobi-Maupertuis metric) over $R$. The pairs $\left(\gamma_{N}(0), \dot{\gamma}_{N}(0)\right.$ lie in this compact space, so we we can find a subsequence of them which converge to some initial condition $(q, v)$. The geodesic with initial condition $(q, v)$ realizes the infinite sequence $s$. This establishes the existence of a solution realizing the syzygy sequence $s$.

QED

\section{FIGURE, SECTION AND EQ RELABELLING NEEDED BELOW XX}

\subsection{Collision sequences. Proof of theorem 3.}

Let $s$ be a collision sequence. We may assume, without loss of generality, that it is a forward collision sequence, and that the two letters in its forward tail are 1 and 2 . The first part of the theorem asserts that any solution which realizes $s$ must satisfy $r_{12} \rightarrow 0$ as the Jacobi time $t \rightarrow \infty$. (In Newtonian time the collision occurs in finite time. As the two bodies get closer, the third body effects them less and less. A straightforward analysis of the two-body $1 / r^{2}$ problem shows that $\lim \inf r_{12}=0$ if and only if $\lim r_{12}=0$. The perturbation of the third body does not affect this assertion. Thus in order to prove the solution realizing $s$ suffers collision it suffices to show that

$$
\liminf r_{12}=0 \quad(6.3 .1)
$$

along the solution.

Our proof of (6.3.1) relies on the fact that on a simply connected complete surface of nonnegative curvature any compact geodesic arc is the unique minimizer between its endpoints. On a complete non-simply connected surface such as the pair of pants this implies that if we have a geodesic arc, then there is no shorter curve which share endpoints with that arc and which is homotopic to it through endpoint-fixing homotopies.

We argue by contradiction. Suppose that some solution $\gamma \subset P$ realizes the collision sequence $s$ but satisfies $\lim \inf r_{12}=\delta>0$. We will construct a comparison curve $c$ which has the same endpoints as a (long) arc of $\gamma$, is homotopic to this arc through end-point fixing homotopies, but which is shorter than $\gamma$. The arc will be one whose syzygy sequence is $1212 \ldots 12$ with $N$ repeats of 12 , and $N$ large. See figure 6 for the picture of this arc 
and the shorter comparison curve $c$. The existence of $c$ contradicts the minimality of $\gamma$ described in the previous paragraph.

To construct $c$ we will use the cylindrical coordinates $(\lambda, \chi)$ of $(3.14 \mathrm{a}, \mathrm{b}),(3.15)$ associated to the 12-collision end. We have

$$
\lambda(\rho, \chi)=\int_{\rho_{0}}^{\rho} \sqrt{U(s, \chi)} d s
$$

where $(\rho, \chi)$ are spherical-polar coordinates centered at the collision. The $(\lambda, \chi)$ coordinates are valid on the entire sphere minus the ' 3 ' equatorial arc and the collision points. The coordinate $\lambda$ satifies $\lambda \rightarrow \infty$ as collision is approached. The Jacobi metric in these coordinates is

$$
d s^{2}=d \lambda^{2}+f(\lambda, \chi)^{2} d \chi^{2}
$$

where

$$
f^{2}=\frac{1}{2} \cos ^{2}(\rho)\left\{1+\frac{s_{12}}{s_{13}}+\frac{s_{12}}{s_{23}}\right\}
$$

The curvature of any metric of the form (6.3.2) is $-\frac{1}{f} \frac{\partial^{2} f}{\partial \lambda^{2}}$. Since this curvature is negative (theorem 2) and since $\lim _{\lambda \rightarrow \infty} f(\lambda, \chi)=1 / \sqrt{2}$ (eq. 3.18) we have that for each fixed $\chi$ the function $f(\chi, \lambda)$ decreases monotonically to its infimum $1 / \sqrt{2}$ as $\lambda \rightarrow \infty$. It follows that $F(\lambda)=\min _{\chi} f(\lambda, \chi)$ also decreases monotonically to $1 / \sqrt{2}$.

Any geodesic arc $\gamma$ on the pair of pants which realizes the syzygy sequence 12 cannot cross either isosceles circle $r_{12}=r_{23}$ or $r_{12}=r_{13}$. This follows from the minimality property of the arc, discussed above, and the reflection principle (as in $[\mathrm{ChM}]$ and the proof of no stuttering in section 2 , between theorems 3 and 4 ). Reflections about the isosceles circles are isometries of the Jacobi metric, so that any segment of $\gamma$ which crosses, then crosses back, can be reflected, so as to form a new arc with the same endpoints as $\gamma$, and the same homotopy type, contradicting uniqueness. Thus, without loss of generality, we may assume that our long subarc of $\gamma$ lies entirely in the union of the regions $r_{12}<r_{13}$ and $r_{12}<r_{23}$. In particular, the $(\lambda, \chi)$ coordinates are valid all along our arc.

To construct the desired comparison curve $c$ we will use the fact that $F$ is monotone decreasing in $\lambda$ and that the number $N$ of 12 crossings of a subarc of $\gamma$ can be taken arbitrarily large. Since inf $r_{12}>0$, by assumption, we have that $\Lambda=\sup \lambda<\infty$ along our curve. Let $\epsilon>0$ small be given. Choose points $\gamma\left(s_{N}\right), \gamma\left(t_{N}\right) s_{N}<t_{N}$, along the arc for which $\lambda\left(t_{n}\right), \lambda\left(s_{n}\right)>\Lambda-\epsilon$ and such that the arc $\gamma\left[s_{n}, t_{n}\right]$ in between realizes the syzygy sequence $12 \ldots 12$ with $N$ copies of 12 . Our comparison curve $c$ will go "straight in" to collision until some point with $\lambda_{*}>\Lambda$, to be determined momentarily, winds around the 12 collision point in the same sense as $\gamma$ for the same number of syzygies at this fixed value $\lambda=\lambda_{*}$, and then return headed "straight out" from collision to the point $\gamma\left(t_{n}\right)$. See figure 6. "Straight in" and "straight out" means that $\chi$ is fixed, and only $\lambda$ varies. During its 'winding around' journey, $\lambda=\lambda_{*}$ is fixed and $\chi$ varies, starting at $\chi=\chi\left(\gamma\left(s_{n}\right)\right)$, increasing so that $c$ suffers the syzygy sequence $1212 \ldots 12$ ( $\mathrm{N}$ times), and then stopping at $\chi=\chi\left(\gamma\left(t_{n}\right)\right.$ in time for the "straight out" return segment. 


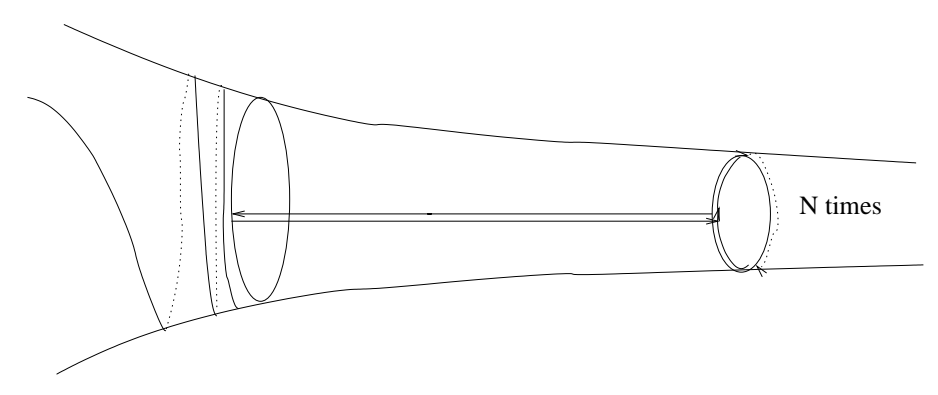

Figure 6. Orbit surgery to shorten length of a collision sequence path FFXX LABEL FIG as per next to last par of this sec.

By construction, the curve $c$ shares endpoints with our arc of $\gamma$, and is homotopic to it. It remains to show that $c$ is shorter than our arc of $\gamma$. By the monotonicity and the limiting properties of $f$ we can choose $\lambda_{*}>\Lambda$ so that

$$
\max _{\chi} f\left(\lambda_{*}, \chi\right)<F(\Lambda):=\min _{\chi} f(\Lambda, \chi)
$$

Choose $N$ so large that

$$
\frac{2\left(\lambda_{*}-\Lambda\right)+\epsilon+\pi F(\Lambda)}{N}<\pi\left(F(\Lambda)-\max _{\chi} f\left(\lambda_{*}, \chi\right)\right) .
$$

It follows that

$$
\pi N \max _{\chi} f\left(\lambda_{*}, \chi\right)+\pi \max _{\chi} f\left(\lambda_{*}, \chi\right)+2\left(\lambda_{*}-\Lambda\right)+2 \epsilon<N \pi(F(\Lambda)
$$

Now consider the cartoon in figure XX or the construction of $c$. The 'in and out' arcs of $c$ have length less than $2\left(\lambda_{*}-\Lambda\right)+2 \epsilon$. The 'around arc' between the $N$ syzyygies has length less than $\pi N \max _{\chi} f\left(\lambda_{*}, \chi\right)$. (Refer again to figure 6.) The additional $\pi \max _{\chi} f\left(\lambda_{*}, \chi\right)$ accounts for the fact that $\chi\left(s_{n}\right)$ and $\chi\left(t_{n}\right)$ need not be equal. Thus the left hand side of the inequality is greater than the length of $c$. A similar but simpler analysis shows that the right hand side is smaller than the length of our arc of $\gamma$. Thus $c$ is shorter than the arc of $\gamma$, completing the proof of first assertion of theorem 3 .

The same analysis shows that the infinite sequence . . 1212 .. of all 12's is never realized. For such a realization must be a local minimizer, and the above orbit surgery shows we can always decrease the length of a path by making it closer to collision.

QED

\subsection{Proof of theorem 4.}

To establish the uniqueness of the realizing solutions, we work on the universal cover $D$ of the pair of pants $P$. Topologically, $D$ is the Poincare disc and the fundamental group 
$\Gamma=\pi_{1}(P)$ (the free group on two letters) acts on $D$ as a Fuchsian group. See figure 3, and also the book Indra's Pearls [Mumf]. In figure $3 \mathrm{~b}$ we have drawn in a fundamental domain $P_{0}$ for $P$ and some of its images under $\Gamma$. The boundary of $P_{0}$ consists of four circular arcs which are lines lines relative to the Poincare metric on $D$. These bounding arcs are labelled $1_{+}, 1_{-}$and $2_{+}, 2_{-}$. To form $P$ out of $P_{0}$ glue arcs $1_{+}$and $1_{-}$to form syzygy arc 1 , and glue arcs $2_{+}$and $2_{-}$to form syzygy arc 2 . Syzygy arc 3 is internal to the fundamental domain. In the figure we have dropped the,+- subscripts on the arcs. 


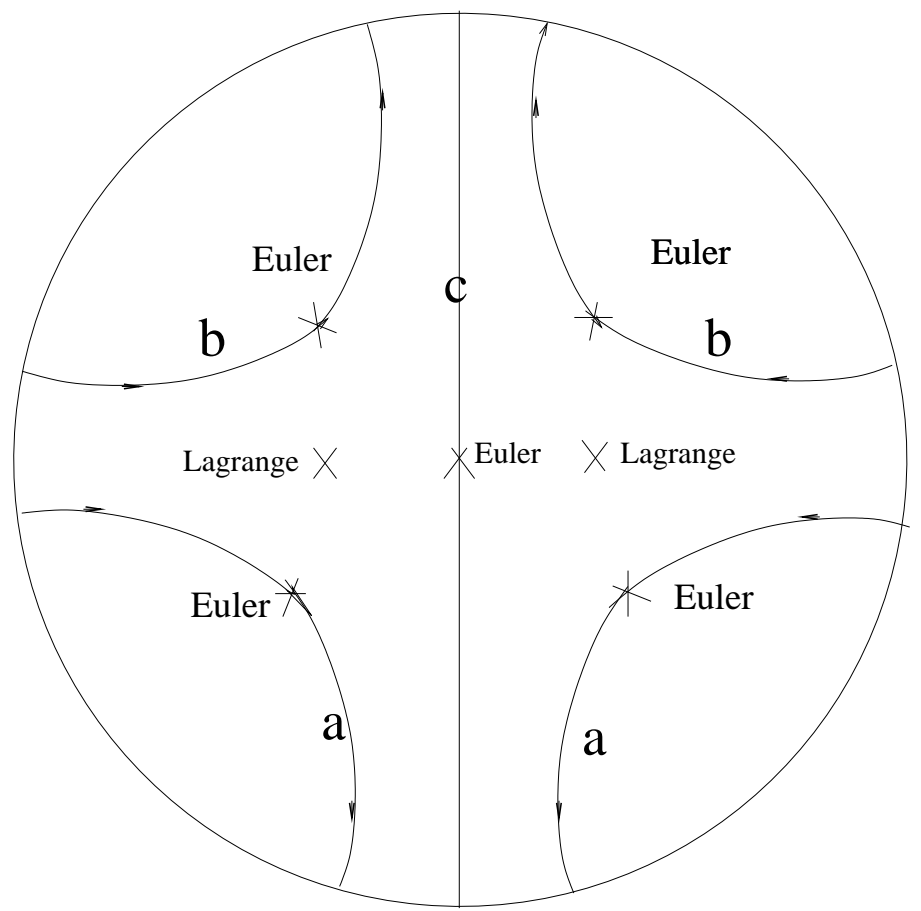

Figure 3a. The fundamental domain

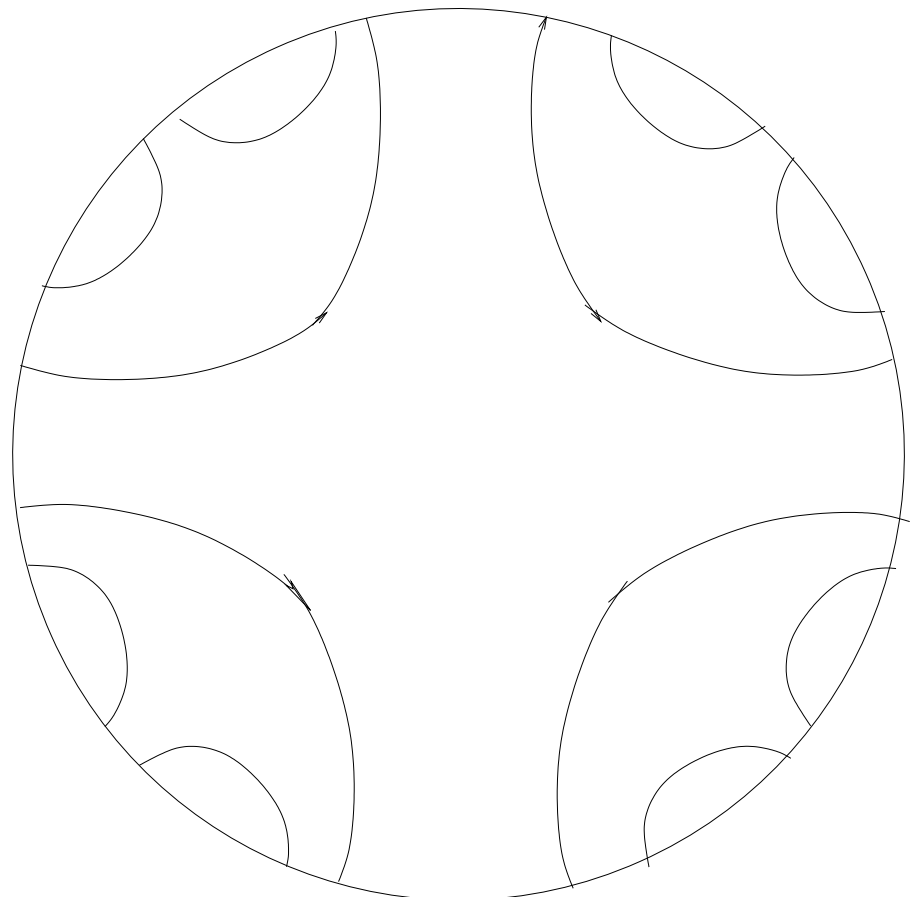

Figure 3b. Some of the tiles.

Write

$$
\pi: D \rightarrow P
$$


for the covering map, so that the fibers of $\pi$ are copies of $\Gamma$. We will use the hyperbolic, or constant negative curvature metric on $D$ in order to understand the fundamental group $\Gamma$ and its action on $D$. (With respect to this metric $\pi$ is not a local isometry.) $\Gamma$ acts on $D$ with respect to hyperbolic isometries, i.e. Möbius transformations. $\Gamma$ is freely generated by two elements $a$ and $b$, one of which, say $a$, interchanges $1_{+}$and $1_{-}$, and the other of which, $b$, interchanges $2_{+}$and $2_{-}$. These elements act on $D$ as Mobius transformations. (Viewed as acting on the Riemann sphere, they interchange exteriors with interiors of their respective circles. ) In order to form $P$ out of $P_{0}$ glue arc $1^{+}$to $1_{-}$by $a$ and glue $2_{+}$to $2_{-}$by $b$. The projection under $\pi$ of these boundary arcs form the arcs 1,2 of the equator of $P$. The third arc 3 is internal to $P_{0}$, and separates it into two halves, the northern $(+)$ hemisphere, and southern (-) hemisphere.

The images of $\gamma P_{0}$ of the fundamental domain $P_{0}$ under elements of $\gamma \in \Gamma$ tile all of $D$. Each of the four boundary arcs of such a tile $\gamma P$ is the image under $\gamma$ of a unique boundary arc of $P_{0}$ and we continue to label the tile's boundary arcs by the corresponding $P_{0}$ lables $1_{+}, 1_{-}, 2_{+}$, or $2_{-}$. Two tiles intersect, if at all, along a common boundary arc. This common arc must be a $j_{-}$arc of one tile and a $j_{+}$of the other, $j=1,2$.

Suppose now that two geodesics realize the same symbol sequence $s$. Denote by $\gamma, c$ the lifts of these geodesics to the universal cover $D$. After translating these curves by elements of $\Gamma$ we may suppose that both begin in the reference fundamental domain $P_{0}$. I claim that the syzygy sequence $s$ uniquely specificies a sequence of contiguous tiles $\ldots P_{-2} P_{-1} P_{0} P_{1} P_{2} \ldots$ through which $c$ and $\gamma$ must pass. To see this fact, we first note that each sequence of three contiguous tiles $P_{i-1} P_{i} P_{i+1}$ represents either two or three letters of a signed syzygy sequence. See figure 4 . The sequence is obtained by drawing a curve which crosses from $P_{i-1}$ through $P_{i}$ and into $P_{i+1}$ in the "most direct" way. The curve must enter into $P_{i}$ across one of its bounding arcs $1_{+}, 1_{-}, 2_{+}, 2_{-}$. The choice of $P_{i-1}$ uniquely specifies which arc. It must leave across another such arc and the choice of $P_{i+1}$ uniquely specifies this exit arc. Along the way it must either cross 3 or not. If no internal syzygy with arc 3 occurs then the sequence has two letters and no ' 3 ', and otherwise the sequence does contain the letter 3 as the middle letter. Consequently, both $\gamma, c$ pass through an identical list of tiling domains, as claimed.

Each tiling domain $P_{j}$ has within it an inverse image $R_{j}=\pi^{-1}(R) \cap P_{j}$ of our compact domain $R$. By lemma 6.1 both $c$ and $\gamma$ must have the property that for infinitely many $j$ we have that both $\gamma$ and $c$ lie in $R_{j}$. 


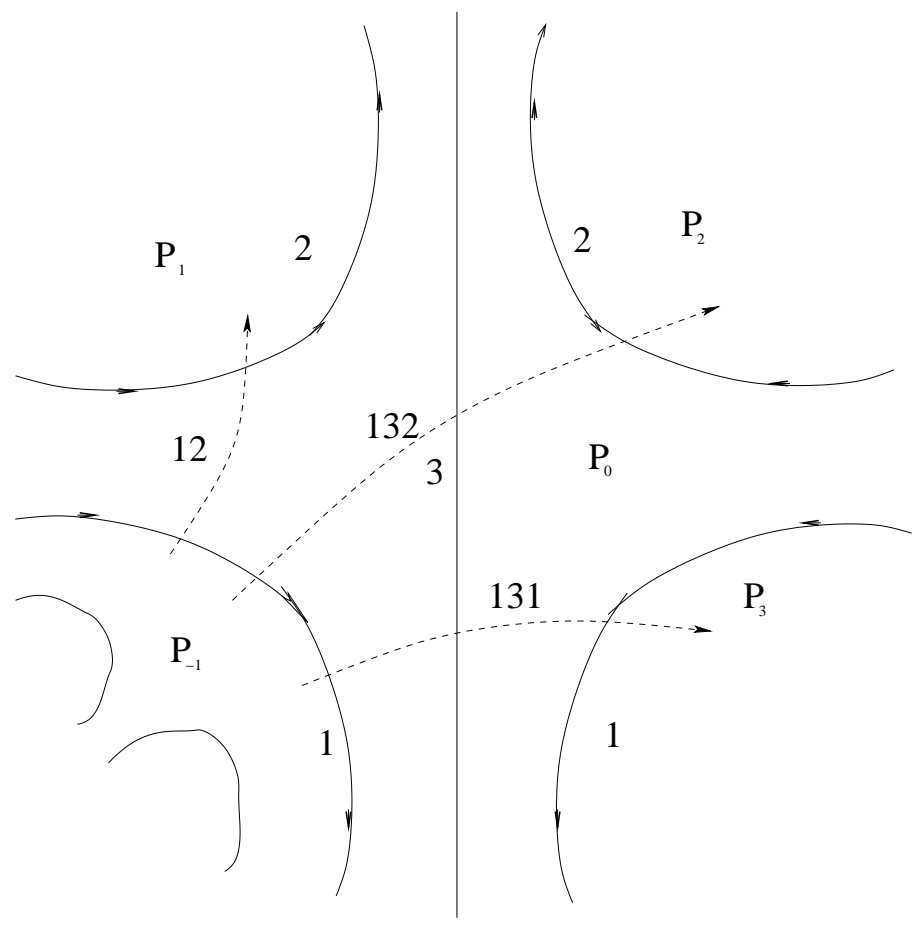

Figure 4. Syzygies and Tile Crossings.

Now lift the Jacobi-Maupertuis metric from $P$ up to $D$, using the projection $\pi: D \rightarrow P$, thus arriving at a complete non-negatively curved $\Gamma$-invariant metric on $D$ for which our two curves $\gamma$ and $c$ are geodesics. The curvature of this metric is zero only at the discrete set of points $\pi^{-1}\left(L_{ \pm}\right)$, where $L_{ \pm}$are the Lagrange points of $P$. And the map $\pi$ is a local isometry for this metric. Write

$$
h(t)=\operatorname{dist}(\gamma, c(t))
$$

for the distance between the variable point $c(t)$ on the curve $c$ and the entire geodesic $\gamma$. See figure 8. Now each $R_{j}$ has finite diameter $\delta$ because $R$ is compact, and the two curves pass through the $R_{j}$ 's infinitely often, indeed every time the letters 123 occurs contiguously in $s$. It follows that

$$
\lim \inf _{t \rightarrow+\infty} h(t) \leq \delta \text { and } \lim \inf _{t \rightarrow-\infty} h(t) \leq \delta .
$$

We will now show that inequality (6.4.2) is impossible unless the two geodesics are in fact the same, in which case $h(t)=0$ everywhere. We use the formula

$$
d^{2} h / d t^{2}=-\sin (A(t)) \int_{d_{t}} K d s
$$

proved in the following paragraph. In this formula, $d_{t}$ is the geodesic realizing the distance $h(t)$. It has one endpoint at the point $c(t)$ on $c$ and the other endpoint on $\gamma$ which it 
intersects perpindicularly. See figure 5. The angle $A(t)$ is the angle of intersection between the geodesics $c$ and $d_{t}$ at $c(t)$. See figure 5. This angle satisfies $0<A(t)<\pi$, so that $\sin (A(t)>0$ The negativity of $K$ (except at a discrete point set) implies that $h(t)$ is strictly convex: $d^{2} h / d t^{2}>0$. But any strictly convex function defined on the real line tends to infinity in one direction or the other. This contradicts (6.4.2). Our two geodesics must be the same.

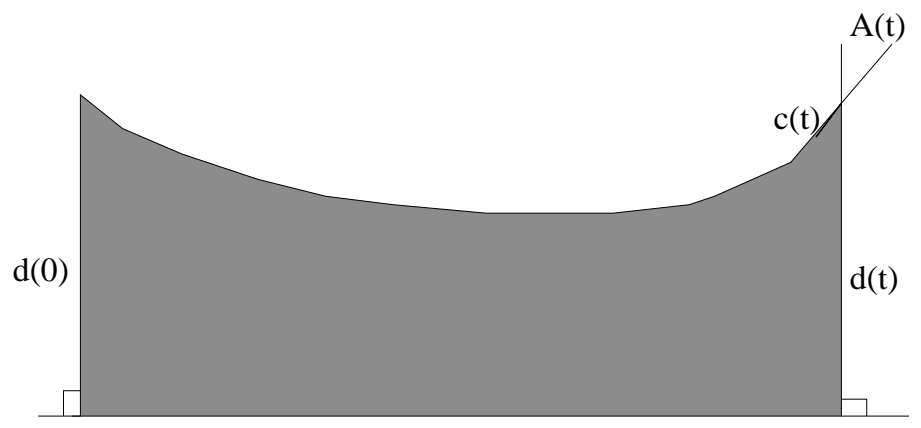

Figure 5. Variation of Distance

\section{Derivation of (6.4.3).}

The first variation of arclength implies

$$
d h / d t=\cos (A(t))
$$

Let $M(t) \subset D$ denote the quadrilateral whose edges consist of the geodesic arcs $d_{0}, d_{t}$ together with the arcs of $\gamma, c$ which connect $d_{0}$ to $d_{t}$. According to the Gauss-Bonnet theorem, for any such geodesic quadrilateral $Q$ we have

$$
2 \pi-(\Sigma \text { interior angles })=-\iint_{Q} K d A
$$

In the case of $M(t)$ the interior angles are $\pi / 2, \pi / 2, \pi-A(0)$ and $A(t)$. See figure 5 again. Thus

$$
A(0)-A(t)=-\iint_{M(t)} K(t) d A
$$

Differentiating (6.4.4) with respect to $t$ yields

$$
d A / d t=\int_{d_{t}} K d s
$$

Now differentiate (6.4.4) with respect to $t$, using (6.4.6) to obtain (6.4.3).

Proof of theorem 5. Let $c$ be a bound orbit and $s=\left\{s_{j}\right\}_{j=-\infty}^{\infty}$ its syzygy sequence. Approximate $s$ by a family $w^{n}, n=1,2,3, \ldots$ of forward collision sequences by replacing 
the tails $s_{j}, j>n$ of $s$ by that of the 12 collision sequence. Collapse two letters if neccessary when stutters appear at the 'join' $j=n$ of the replacement. The backward tails of the $w^{n}$ contain all three letters because $s$ is collision-free, so we can apply theorem 2 to realize the $w^{n}$ by solutions $\gamma_{n}$ (not necessarily unique). By lemma 6.1 the $\gamma_{n}$ all pass through $R$, so, shifting time if necessary, we have that the tangent vectors $v_{n}=\left(\gamma_{n}(0), \dot{\gamma}_{n}(0)\right)$ are unit vectors with position $\gamma_{n}(0)$ in $R$. We now argue as in the proof of theorem 3. By compactness of the set of unit tangent vectors over $R$, we can form a convergent subsequence of the $v_{n}$, which we relabel as $v_{n}$, so that $v_{n} \rightarrow v$. Let $\gamma$ be the curve with initial condition $v$. The curves $\gamma_{n}$ converge, over compact sets, to $\gamma$, so that the syzygy sequences $w^{n}$ must converge to the syzygy sequence of $\gamma$. Thus $\gamma$ and $c$ share the same syzygy sequence. By theorem $4, \gamma=c$. Consequently the unboounded curves $\gamma_{n}$ converge to our initial bound curve $c$.

QED

\section{Curvature for unequal masses.}

In this section is to prove that Theorem 1 is a special case: for most mass distributions the curvature takes on both signs. Take the masses to be general positive numbers $m_{i}$. Writing

$$
p_{i}=m_{j} m_{k}, s_{i}=r_{j k}^{2}
$$

for $i j k$ a permutation of 123 we have

$$
U=\Sigma p_{i} / s_{i}, \quad I=\frac{1}{M} \Sigma p_{i} s_{i}, \text { where } M=\Sigma m_{i} .
$$

The Jacobi metric is obtained by multiplying the shape metric $d s_{m}^{2}$ on the shape sphere $I=1$ by $U$ and restricting it to the sphere $I=1$. (The subscript ' $\mathrm{m}$ ' indicates dependence on the masses.) It is conceptually and computationally more straightforward to identify the shape sphere as the space of rays in shape space and to make $U$ a function on the space of rays by making it homogeneous of degree 0 by multiplying it by $I$. Thus, setting

$$
\tilde{U}=I U
$$

our Jacobi metric is

$$
d s_{J}^{2}=\tilde{U} d s_{m}^{2} .
$$

In order to compute, we will use the coordinates $\phi, \theta, R$ of [Mont2] for shape space. (See, in particular, the notation and computations of section 7 there.) Write $I_{1}$ for the moment of inertial when all masses are equal to one:

$$
I_{1}=\Sigma r_{i j}^{2} / 3
$$

The $\phi, \theta$ are spherical coordinates for the $I_{1}$ shape sphere, while the radial coordinate $R=\sqrt{I}$ for the mass-dependent $I$ of (3.6). With respect to these coordinates we have that

$$
s_{k}=I_{1}\left(1-\gamma_{k}(\theta) \cos (\phi)\right.
$$


as before Note that the coordinates $\theta, \phi$ and the functions $s_{k}$ do not vary as the masses are changes. The metric $d s_{m}^{2}$ when expressed in our coordinates is mass-dependent and is given by

$$
d s_{m}^{2}=\lambda^{2} d s_{1}^{2}
$$

where

$$
d s_{1}^{2}=\frac{1}{4}\left(d \phi^{2}+\cos (\phi)^{2} d \theta^{2}\right)
$$

(see [MontI], eq (5.6) and Prop. 2) is the shape sphere metric when all the masses are 1, and where the conformal factor $\lambda$ is given by

$$
\lambda=d(m) I_{1} / I ; \quad d(m)=\sqrt{3 m_{1} m_{2} m_{3} / M}
$$

(See eq (5.7) of [MontI]). The metric we are to work with is thus

$$
d s_{J}^{2}=\tilde{U} \lambda^{2} d s_{1}^{2}
$$

Its curvature is given by lemma 4.1 :

$$
\bar{K}=\frac{1}{\tilde{U} \lambda^{2}}\left\{4-\frac{1}{2} \Delta \log \left(\tilde{U} \lambda^{2}\right)\right\}
$$

where the Laplacian $\Delta$ is with respect to the metric $d s_{1}^{2}$. The conformal factor is

$$
\begin{aligned}
\tilde{U} \lambda^{2} & =d(m)^{2} I U I_{1}^{2} / I^{2} \\
& =d(m) I_{1} U\left(d(m) I_{1} / I\right) \\
& =d(m) \hat{U} \lambda
\end{aligned}
$$

where

$$
\hat{U}=I_{1} U
$$

Then

$$
\Delta \log \tilde{U} \lambda^{2}=\Delta \log \hat{U}+\Delta \log \lambda
$$

Set

$$
\hat{s}_{i}=s_{i} / I_{1}=\left(1-\gamma_{k}(\theta) \cos (\phi)\right.
$$

so that

$$
\hat{U}=\Sigma p_{i} / \hat{s}_{i}
$$

Since $\hat{s}_{i}$ is equal to the variable $s_{i}$ as given by eq. $(5.1 .1 \mathrm{~b})$, and since the Laplacian is the Laplacian of that section, we can continue to compute as per section 5 . We have

$$
\hat{U} \lambda \bar{K}=4-\frac{1}{2} \Delta \log (\hat{U})-\frac{1}{2} \Delta \log (\lambda)
$$


To compute the last term $-\frac{1}{2} \Delta \log (\lambda)$ of $(7.5)$ we use the fact that the metrics $d s_{m}^{2}$ are $d s_{1}^{2}$ through $\lambda^{2}$, and that both have curvature 4 . From lemma 4.1 it follows that

$$
4=\frac{1}{\lambda^{2}}\left(4-\frac{1}{2} \Delta \log \lambda^{2}\right\}
$$

or

$$
4 \lambda^{2}-4=-\Delta \log \lambda
$$

Equation (7.5) can then be rewritten

$$
-\hat{U}^{3} \lambda \bar{K}=-\hat{U}^{2}\left(4-\frac{1}{2} \Delta \log (\hat{U})\right)+\hat{U}^{2}\left(2-2 \lambda^{2}\right)
$$

Computation of $\Delta \hat{U}$. To ease notation, we drop the hats for this subsection, so that $U$ means the function $\hat{U}$ and the $s_{k}$ means the function $\hat{s}_{k}$. The structural form of all the formulae and calculations of section 5 remains intact provided we insert the weightings $p_{i}$ in the correct places.

We proceed to the computation of $\Delta \log (\hat{U}))$. Equations (4.2) and (5.2.1) continue to hold with $\hat{U}$ in place of $U$, and equations $(5.2 .2 \mathrm{a}, \mathrm{b})$ hold with $\hat{s}_{i}$ in place of $s_{i}$. The analogues of $(5.2 .3),(5.2 .4)$ are

$$
\frac{1}{c} \partial_{\phi}\left(c \partial_{\phi} U\right)=\left(\left(s^{2}-c^{2}\right) / c\right) \Sigma p_{i} \gamma_{i} / s_{i}^{2}+2 s^{2} \Sigma p_{i} \gamma_{i}^{2} / s_{i}^{3}
$$

And

$$
\frac{1}{c^{2}} \partial_{\theta} \partial_{\theta} U=\frac{1}{c} \Sigma p_{i} \gamma_{i} \prime \prime / s_{i}^{2}+2 \Sigma p_{i}\left(\gamma_{i}^{\prime}\right)^{2} / s_{i}^{3}
$$

Now note that algebraic steps going from (5.2.5) to (5.2.7) apply term by term, so can be carried over verbatim except that the $i$ th term must be multiplied by $p_{i}$. Thus (reverting to hats)

$$
\Delta \hat{U}=8 \hat{U}_{4}
$$

where

$$
\hat{U}_{4}=\Sigma p_{i} / \hat{s}_{i}^{2}
$$

The first line of (5.3.2) becomes

$$
\|\nabla U\|^{2}=4\left(\Sigma s p_{i} \gamma_{i} / s_{i}^{2}\right)^{2}+4\left(\Sigma p_{i} \gamma_{i}^{\prime} / s_{i}^{2}\right)^{2}
$$

The algebra which follows is essentially the same, leading to

$$
\|\nabla U\|^{2}=4 S
$$

where

$$
S=2 \Sigma p_{i}^{2} / s_{i}^{3}-\Sigma p_{i}^{2} / s_{i}^{3}-3 / 2 \Sigma^{\prime} p_{i} p_{j} / s_{i}^{2} s_{j}^{2}+2 \Sigma^{\prime} p_{i} p_{j} / s_{i} s_{j}^{2}-\Sigma^{\prime} p_{i} p_{j} / s_{i} s_{j}
$$


Combining (7.8) and (7.9) according to (4.2) (see also the steps (4.7)-(4.9)) yields the formula:

$$
-U^{2}\left(4-\frac{1}{2} \Delta \log U\right)=3 \Sigma^{\prime} p_{i} p_{j} / s_{i}^{2} s_{j}^{2}-2\left(\Sigma p_{i} / s_{i}\right)^{2}
$$

From earlier, we have

$$
U^{2}\left(2-2 \lambda^{2}\right)=2\left(\Sigma p_{i} / s_{i}\right)^{2}-2\left(\Sigma p_{i} / s_{i}\right)^{2} d(m)^{2}\left(\frac{1}{3} \Sigma s_{i}\right)^{2} /\left(\Sigma p_{i} s_{i} / M\right)^{2},
$$

(We continue to use $s_{i}$ in place of $\hat{s}_{i}$.) Upon adding (7.10) and (7.11) there is a cancellation yielding:

$$
-U^{3} \lambda^{2} \bar{K}=3 \Sigma^{\prime} p_{i} p_{j} / s_{i}^{2} s_{j}^{2}-2\left(\Sigma p_{i} / s_{i}\right)^{2} d(m)^{2}\left(\frac{1}{3} \Sigma s_{i}\right)^{2} /\left(\Sigma p_{i} s_{i} / M\right)^{2} .
$$

A computation shows that

$$
d(m)^{2} M^{2}=\frac{3}{2} \Sigma^{\prime} p_{i} p_{j}
$$

Recall that the $s_{i}$ (the previous $\hat{s}_{i}$ 's) satisfy $\frac{1}{3} \Sigma s_{i}=1$. We finally obtain:

$$
-U^{3} \lambda^{2} \bar{K}=3\left\{\Sigma^{\prime} p_{i} p_{j} / s_{i}^{2} s_{j}^{2}-\Sigma^{\prime} p_{i} p_{j} \frac{\left(\Sigma p_{i} / s_{i}\right)^{2}}{\left(\Sigma p_{i} s_{i}\right)^{2}}\right\}
$$

Consequently

$$
\kappa=\sqrt{\Sigma^{\prime} \frac{p_{i} p_{j}}{s_{i}^{2} s_{j}^{2}}} \frac{\left(\Sigma p_{i} s_{i}\right)}{\left(\Sigma p_{i} / s_{i}\right)}-\sqrt{\Sigma^{\prime} p_{i} p_{j}}
$$

governs the sign of the curvature, with the curvature $\bar{K}$ negative if $\kappa$ is positive, positive if $\kappa$ is negative, and zero if $\kappa$ is zero. We note that $\kappa$, and hence the curvature is zero at the Lagrange point $s_{1}=s_{2}=s_{3}=1$, and that this is true for all choices of the masses $p_{i}$.

Theorem 6. For a Zariski-dense set of mass distributions, the sign of the curvature changes in a neighborhood of the Lagrange point.

Proof. It suffices to show that for a Zariski-dense set of mass distributions the differential $d \kappa \neq 0$ at the Lagrange point. A differential form $\Sigma a_{i} d s_{i}$ represents zero on the shape sphere if and only if it is proportional to $\Sigma d s_{i}$, the latter being the differential of the constraint $\Sigma s_{i}=3$ satisfied by the $s_{i}$ (which are the old $\hat{s}_{i}$ 's). A computation shows that at the Lagrange point $s_{i}=1$ we have

$$
\beta d \kappa=p_{1}\left(p_{2}^{2}+p_{3}^{2}\right) d s_{1}+p_{2}\left(p_{1}^{2}+p_{3}^{2}\right) d s_{2}+p_{3}\left(p_{1}^{2}+p_{2}^{2}\right) d s_{3} \bmod \Sigma d s_{i}
$$

where $\beta$ is a nonzero constant. We thus want to know whether or not the equality

$$
\left(p_{1}\left(p_{2}^{2}+p_{3}^{2}\right), p_{2}\left(p_{1}^{2}+p_{3}^{2}\right), p_{3}\left(p_{1}^{2}+p_{2}^{2}\right)\right)=(\lambda, \lambda, \lambda)
$$


can be satisfied for some $\lambda$. The right hand side of equation (**), being homogeneous of degree 3 , defines a polynomial map $\mathbb{R} \mathbb{P}^{2} \rightarrow \mathbb{R} \mathbb{P}^{2}$ and we want to know if it is equal to the constant map $[1,1,1]$. Because the map is polynomial, if we can exhibit a single point where the inequality fails then it must fail on a Zariski-dense set. Plugging in $p_{1}=p_{2}=1, p_{3}=a$ yields $\left(p_{1}\left(p_{2}^{2}+p_{3}^{2}\right), p_{2}\left(p_{1}^{2}+p_{3}^{2}\right), p_{3}\left(p_{1}^{2}+p_{2}^{2}\right)\right)=\left(1+a^{2}, 1+a^{2}, 2 a\right)$ which is not proportional to $(1,1,1)$ unless $a=1$.

QED

\section{Appendix A}

We prove

Theorem A. The set of initial conditions within $H=0, I=1, c=0$ whose solutions tend to a binary collision of type $i j$ has nonempty interior. This fact holds for all positive mass distributions.

Proof of theorem A.We use Newtonian time and Jacobi coordinates For notational simplicity, take $i j=12$. The Jacobi coordinates are $\zeta_{1}=x_{1}-x_{2}, \zeta_{2}=x_{3}-\left(m_{1} x_{1}+\right.$ $\left.m_{2} x_{2}\right) /\left(m_{1}+m_{2}\right)$. The distance to binary collision is

$$
r=\left|\zeta_{1}\right|
$$

We will exhibit a nonempty open set of initial conditions at time $t=0$ for which $r(t)=0$ for some time $t<O(r(0))$.

The Hamiltonian is

$$
H=\frac{1}{2}\left(\mu_{1}\left|\dot{\zeta}_{1}\right|^{2}+\mu_{2}\left|\dot{\zeta}_{2}\right|^{2}\right)-\frac{m_{1} m_{2}}{r^{2}}-W\left(\zeta_{1}, \zeta_{2}\right)
$$

where $\mu_{1}=m_{1} m_{2} /\left(m_{1}+m_{2}\right), \mu_{2}=m_{3}\left(m_{1}+m_{2}\right) / M, W=m_{1} m_{3} / s_{2}+m_{2} m_{3} / s_{1}$ and the squared distances $s_{2}, s_{3}$ can be expressed $\left|\zeta_{2}+a_{i} \zeta_{1}\right|^{2}$ in Jacobi coordinates, with mass-dependent nonzero constants $a_{i}$. The interaction term $W$ satisfies the estimates

$$
\begin{array}{r}
|W| \leq C_{1}+C_{2} \epsilon, \quad \text { for } r<\epsilon \quad(3 A), \\
\left|\frac{\partial W}{\partial \zeta_{1}}\right| \leq C_{1}+C_{2} \epsilon,\left|\frac{\partial W}{\partial \zeta_{2}}\right| \leq C_{1}+C_{2} \epsilon, \quad \text { for } r \leq \epsilon
\end{array}
$$

( $\epsilon$ sufficiently small), where $C_{1}, C_{2}$ are constants depending only on the masses.

The equations of motion are

$$
\ddot{\zeta}_{1}=-\left(m_{1}+m_{2}\right) \frac{\zeta_{1}}{r^{4}}+\frac{1}{\mu_{1}} \frac{\partial W}{\partial \zeta_{1}}
$$

and

$$
\ddot{\zeta}_{2}=\frac{1}{\mu_{2}} \frac{\partial W}{\partial \zeta_{2}}
$$

Write

$$
J_{1}=\zeta_{1} \wedge \dot{\zeta}_{1}
$$


for the angular momentum (up to a factor of $\mu_{1}$ ) of the 12 system. We compute

$$
\begin{aligned}
\dot{J}_{1} & =\zeta_{1} \wedge \ddot{\zeta}_{1} \\
& =\zeta_{1} \wedge \frac{1}{\mu_{1}} \frac{\partial W}{\partial \zeta_{1}}
\end{aligned}
$$

so that

$$
\left|\dot{J}_{1}\right| \leq C r
$$

Because $\left|\dot{\zeta}_{1}\right|^{2}=\dot{r}^{2}+J_{1}^{2} / r^{2}$ we have that

$$
r^{2} H=\frac{1}{2}\left(\mu_{1} r^{2} \dot{r}^{2}+\mu_{1} J_{1}^{2}\right)-m_{1} m_{2}+r^{2}\left(\frac{1}{2} \mu_{2}\left|\dot{\zeta}_{2}\right|^{2}-W\right)
$$

Now let $\zeta(t)=\left(\zeta_{1}(t), \zeta_{2}(t)\right)$ be a solution satisfying the initial conditions $r(0)<\epsilon, H=$ $0, I=1, J=0$. From (6A) and (4A) we have that

$$
\left|\dot{\zeta}_{2}(t)\right|^{2} \leq\left|\dot{\zeta}_{2}(0)\right|^{2}+C t
$$

for $t=O(1)$, provided $r(0)<\epsilon$. Here $C$ depends only on the masses and $\epsilon$. Letting $r \rightarrow 0$, we see from $(9 \mathrm{~A})$ that if our solution is to have a collision then we must have

$$
\lim r^{2} \dot{r}^{2}+\lim J_{1}^{2}-2\left(m_{1}+m_{2}\right)=0,
$$

where we have used $m_{1} m_{2} / \mu_{1}=m_{1}+m_{2}$. But $r^{2} \dot{r}^{2} \geq 0$, so we must have

$$
2\left(m_{1}+m_{2}\right)-\lim J_{1}^{2} \geq 0
$$

We argue in the reverse. Suppose that $2\left(m_{1}+m_{2}\right)-J_{1}(0)^{2}$ is sufficiently positive at the initial time $t=0$, and that $\dot{r}(0)<0$ then (11A) forces $r^{2} \dot{r}^{2}$ to be positive over a finite time interval. We will show that, upon integration, this will force $r(t)=0$ in some finite time $t=O(\sqrt{r(0)})$. Note from the bounds $(10 \mathrm{~A})$ and the fact that $H=0$ we have

$$
\left.\mid \mu_{1} r^{2} \dot{r}^{2}+\mu_{1} J_{1}(0)^{2}\right)-2 m_{1} m_{2} \mid \leq K r(0)
$$

for $0 \leq t \leq 1$ and for as long as $\dot{r}(t)<0$. Here the constant $K$ depends only on the masses and $\dot{\zeta}_{2}(0)$. Dividing by $\mu_{1}$ and using $m_{1} m_{2} / \mu_{1}=m_{1}+m_{2}$ we arrive at

$$
\left.\mid r^{2} \dot{r}^{2}+J_{1}(0)^{2}\right)-2\left(m_{1}+m_{1}\right) \mid \leq K^{*} r(0)
$$

where $K^{*}=K / \mu_{1}$.

We now impose the open condition

$$
2\left(m_{1}+m_{2}\right)-J_{1}(0)^{2}-K^{*} r(0)>\delta^{2}
$$

on our initial conditions. This will be the open condition of theorem A. The positive constant $\delta$ will be constrained further below. It follows from (15A) and (14A) that

$$
\left.\delta^{2}<2\left(m_{1}+m_{2}\right)-J_{1}(0)^{2}\right)-K^{*} r(0) \leq r^{2} \dot{r}^{2}
$$


(16A) together with $\dot{r}(0)<0$ forces $\dot{r}<0$ throughout the time interval in question. Thus $-r \dot{r}>0$, and so we can take square roots of inequality $(16 \mathrm{~A})$ to obtain

$$
\delta \leq-r \dot{r} \quad(17 A)
$$

Taking negatives and integrating we find that $-\delta t \geq \frac{1}{2} r(t)^{2}-\frac{1}{2} r(0)^{2}$ or

$$
r(0)^{2}-2 \delta t \geq r(t)^{2} \quad(18 A)
$$

This forces $r(t)=0$ for some time $t$ with $t \leq r(0)^{2} / 2 \delta$. In order that the collision time $t$ is $o(1)$ it is sufficient to take $\delta=O(r(0))$.

We have proved that a 12 collision occurs within a time $t=r(0)^{2} / 2 \delta$ for all initial conditions satisfying $(15 \mathrm{~A}), \dot{r}(0)<0$, and $r(0)<\epsilon$, where $\epsilon$ is small enough so that the inequalities $(3 \mathrm{~A}, 4 \mathrm{~A})$ are in force. This set of initial conditions is clearly open. It remains to show that this set is nonempty. Consider the collinear solution having $H=0=J$ and $I=1$. (There are precisely two such solutions, up to time translation and rotation, one for each arc of the equator which ends in the 12 collision.) These solutions satisfy $J_{1}=0$. In this case (15A) reads $m_{1}+m_{2}>K r(0)+\delta$. and so will hold for $r(0)$ small provided only that $\delta<m_{1}+m_{2}$. Since the solution tend to collision it follows that (15A) is eventually in force along the collinear solution, and hence that our set of of initial conditions is nonempty. QED

Acknowledgements. I dedicate this paper to the memory of my father. I acknowledge useful correspondences with Toshiaki Fujiwara, Alain Chenciner, Alain Albouy, and conversations with Anatole Katok, Rafe Mazzeo, and with Jeff Xia for pointing out that theorem 1 combined with an earlier version of theorems 2, 3 and 4 ought to imply theorem 5 .

\section{References}

[AbMar] R. Abraham and J. Marsden, Foundations of Mechanics, Benjamin-Cummings, [1978].

[AlbCh] A. Albouy and A. Chenciner, Le probléme des $n$ corps et les distances mutuelles, Invent Math. 131 (1998), no. 1, 151-184.

[Arn] V.I. Arnol'd, Mathematical Methods of Classical Mechanics, SpringerVerlag, [1989].

[Ban] T. Banachiewitz, Sur un cas particulier du probleme des trois corps, CRAS, Paris, 142, (1906), pp 510-512.

[CGMS] A. Chenciner, J. Gerver, R. Montgomery R. and C. SimóSimple choreographies of $N$ bodies: a preliminary study in Geometry, Mechanics and Dynamics, 287-308, Springer,New York, 2002.

[ChMont] Chenciner A. and Montgomery R. A remarkable periodic solution of the threebody problem in the case of equal masses, Annals of Math., 152, pp. 881-901 (2000)

[FerrTerr] Davide Ferrario and Susanna Terracini On the Existence of Collisionless Equivariant Minimizers for the Classical n-body Problem. Math ArXivs, math-ph 0302022 [2003]. 
[Gordon] W. B. Gordon, A minimizing property of keplerian orbits, American Journal of Mathematics, vol. 99, $n^{0} 5$, 961-971, (1970).

[Fuji] T. Fujiwara, H. Fukuda, A. Kameyama, H. Ozaki, M. Yamada, Synchronised Similar Triangles for Three-Body Orbit with Zero Angular Momentum, arxiv.org/abs/math$\mathrm{ph} / 0404056$.

[Hermann] R. Hermann, On the differential geometry of foliations, Ann. of Math. (2), (1959), 445-457

[Math] Mathematical Society of Japan, Encyclopedic Dictionary of Mathematics, by the Mathematical Society of Japan, ed. by S Iyanga and Y Kawada, translated by K. O. May, The MIT Press, Cambridge, Massachussets, and London, England, [1977]

[MontI]Richard Montgomery, Infinitely Many Syzygies, Archives for Rational Mechanics and Analysis, v. 164 (2002), no. 4, 311-340, 2002.

[MontN] Richard Montgomery, The N-body problem, the braid group, and action-minimizing periodic orbits, Nonlinearity, vol. 11, no. 2, 363-376, 1998.

[MontR] Richard Montgomery, Geometric Phase of the Three-Body Problem, Nonlinearity, vol. 9, no. 5, 1341-1360, 1996.

[Moore] Cris Moore, Braids in Classical Gravity, Physical Review Letters 70, pp. 36753679, (1993).

[Morse] H. M. Morse, A one-to-one representation of geodesics on a surface of negative curvature, Am. J. Math, 43, no. 1, 33-51, 1921.

[Mumf] David Mumford, Caroline Series,David Wright, David Indra's pearls. The vision of Felix Klein. Cambridge University Press, New York, 2002.

[Poin] Poincaré, H. [1896], Sur les solutions périodiques et le principe de moindre action, . C.R.A.S. Paris 123, 915-918. 